\title{
A Class of Singular Integral Operators Associated to Surfaces of Revolution*
}

\author{
Muliang Cao ${ }^{1}$, Huoxiong $\mathrm{Wu}^{2 \#}$ \\ ${ }^{1}$ Guangzhou Branch of Institute of Electronic Technology, PLA Information Engineering University, \\ Guangzhou, China \\ ${ }^{2}$ School of Mathematical Sciences, Xiamen University, Fujian, China \\ E-mail:ml_cao@163.com, "huoxwu@xmu.edu.cn
}

Received July 19, 2011; revised August 10, 2011; accepted August 20, 2011

\begin{abstract}
In this paper, the authors establish the $L^{p}$-mapping properties of a class of singular integral operators along surfaces of revolution with rough kernels. The size condition on the kernels is optimal and much weaker than that for the classical Calderon-Zygmund singular integral operators.
\end{abstract}

Keywords: Singular Integrals, Surface of Revolution, Maximal Operator, Rough Kernel

\section{Introduction and Main Results}

Let $R^{n}, n \geq 2$, be the $\mathrm{n}$-dimensional Euclidean space and $S^{n-1}$ be the unit sphere in $R^{n}$ equipped with the normalized Lebesgue measure $\mathrm{d} \sigma=\mathrm{d} \sigma(\bullet)$. Let

$\Gamma_{\phi}=\left\{y, \phi(|y|) ; \quad y \in R^{n}\right\}$ be the surface of revolution generated by a suitable function $\phi:[0, \infty) \rightarrow R$. For nonzero points $x \in R^{n}$, we denote $x^{\prime}=x /|x|$. Let $\Omega \in L^{1}\left(S^{n-1}\right)$ be a homogeneous function of degree zero on $R^{n}$ and satisfy

$$
\int_{S^{n-1}} \Omega\left(y^{\prime}\right) \mathrm{d} \sigma\left(y^{\prime}\right)=0,
$$

Suppose that $h$ is a radial measurable function. Define the singular integral operator $T_{\phi, h}$ in $R^{n+1}$ along $\Gamma_{\phi}$ by

$$
\begin{aligned}
& T_{\phi, h}(f)\left(x, x_{n+1}\right) \\
& =p \cdot v \cdot \int_{R^{n}} \frac{h(|y|) \Omega\left(y^{\prime}\right)}{|y|^{n}} \times f\left(x-y, x_{n+1}-\phi(|y|)\right) \mathrm{d} y
\end{aligned}
$$

for all $f \in \mathbb{S}^{n+1}$ (the Schwartz function class on $R^{n+1}$ ), where $\left(x, x_{n+1}\right) \in R^{n} \times R=R^{n+1}$.

Operators of the type (1.2) have been studied quite extensively (see [1-13] and therein numerous references). We refer the readers to see Stein-Wainger's report [14, 15] for more background information. In 1996, Kim, Wainger, Wright and Ziesler proved the following result.

Theorem A [10]. Let $\phi \in C^{2}([0, \infty))$ be convex, in-

*The project was supported by the NSF of China (G11071200) and the NSF of Fujian Province of China (No. 2010J01013). creasing and $\phi(0)=0$. Suppose that $\Omega \in C^{\infty}\left(S^{n-1}\right)$ is a homogeneous function of degree zero on $R^{n}$ and satisfies (1.1). Then $T_{\phi, 1}$ is bounded on $L^{p}\left(R^{n+1}\right)$ for $1<p<\infty$.

By a minor modification of the proof in Theorem A, one can show that the conclusion of Theorem A remains valid if the condition $\Omega \in C^{\infty}\left(S^{n-1}\right)$ is replaced by the condition $\Omega \in L^{q}\left(S^{n-1}\right)$ for some $q>1$ (see [16, pp. 372-373], as well as [6]). Subsequently, this result was improved and extended by many authors (see $[1,2,5,6$, 11,12] et al.). In particular, in 2001, Lu, Pan and Yang gave the general theorem as follows.

Theorem B [11]. Let $\phi:[0, \infty) \rightarrow R$ be a continuously differentiable on $[0, \infty)$ and satisfy

$|\phi(t)-\phi(0)| \leq C t^{\alpha}$ for some $\alpha$ and small $t$, where $C$ is a constant independent of $t$. Suppose that

$\Omega \in H^{1}\left(S^{n-1}\right)$ and $h \in L^{\infty}([0, \infty))$. Then $T_{\phi, h}$ is bounded on $L^{p}\left(R^{n+1}\right)$ for $1<p<\infty$, provided that the maximal operator $v_{\phi}$ defined by

$$
v_{\phi}(f)(r, s)=\sup _{j \in \mathbb{Z}} \frac{1}{2^{j}} \int_{2^{j}}^{2^{j+1}} f(r-t, s-\phi(t)) \mathrm{d} t
$$

is bounded on $L^{p}\left(R^{2}\right)$ for $p>1$.

Actually, the condition $h \in L^{\infty}([0, \infty))$ can be weakened to the case:

$$
h \in \Delta_{\gamma}\left(R^{+}\right)=\left\{h ; \sup _{s>0} s^{-1} \int_{0}^{s}|h(t)|^{\gamma} \mathrm{d} t<\infty\right\}, \gamma>1
$$

with $|1 / p-1 / 2|<\min \left\{1 / 2,1 / \gamma^{\prime}\right\} \quad$ (see [9]), and the size condition on $\Omega$ in Theorem B is the best one, so far, 
even if $\phi(t) \equiv 0 \quad$ (see [8]).

On the other hand, for $h \in L^{2}\left(R^{+}, r^{-1} d r\right)$, it is known in [17], if $\phi(t) \equiv 0$, that $T_{\phi, h}$ is bounded on $L^{p}\left(R^{n+1}\right)$ for $1<p<\infty$, provided $\Omega \in L\left(\log ^{+} L\right)^{1 / 2}\left(S^{n-1}\right)$, which is optimal and much weaker than that for the classical Calderon-Zygmund singular integral operators. It should be noted that the spaces $L\left(\log ^{+} L\right)^{1 / 2}\left(S^{n-1}\right)$ and $H^{1}\left(S^{n-1}\right)$ do not include in each other.

Inspired by Al-Salman's work [17], we shall establish the following main result in this paper.

Theorem 1. Let $\phi:[0, \infty) \rightarrow R$ be a suitable function, which ensures that the integral in (1.2) exists in principle-value sense when, say, $f \in \mathbb{S}^{n+1}$ (the Schwartz function class on $\left.R^{n+1}\right)$. Suppose that

$h \in L^{2}\left(R^{+}, r^{-1} d r\right), \Omega$ is a homogenous function of degree zero on $R^{n}$ and satisfies (1.1). If

$\Omega \in L\left(\log ^{+} L\right)^{1 / 2}\left(S^{n-1}\right)$, then

1) $\left\|T_{\phi, h}(f)\right\|_{L^{2}\left(R^{n+1}\right)} \leq C\|f\|_{L^{2}\left(R^{n+1}\right)}$.

2) $\left\|T_{\phi, h}(f)\right\|_{L^{p}\left(R^{n+1}\right)} \leq C\|f\|_{L^{p}\left(R^{n+1}\right)}$,

for $1<p<\infty$, provided that the lower dimensional maximal operator $M_{\phi}$ defined by

$$
M_{\phi}(f)(r, s)=\sup _{R>0} R^{-1} \int_{R / 2}^{R}|f(r-t, s-\phi(t))| \mathrm{d} t
$$

is bounded on $L^{p}\left(R^{2}\right)$ for $1<p<\infty$.

In Theorem 1, if $\phi(t)$ is continuously differentiable on $(0, \infty)$ and satisfy $|\phi(t)-\phi(0)| \leq C t^{\alpha}$ for some $\alpha$ and small $t$, where $C$ is a constant independent of $t$, in particular, if $\phi(t) \in C^{1}([0,1))$, then the integral in (1.2) exists in principle-value sense when, say, $f \in \mathbb{S}^{n+1}$ (the Schwartz function class on $R^{n+1}$ ) (see [11]).

Remark 1. The $L^{p}\left(R^{2}\right)$ boundedness of $M_{\phi}$ is known for many $\phi(t)$ 's. A few prominent examples are as follows:

1) If $\phi(t)$ is a real-valued polynomial, then $M_{\phi}$ is bounded on $L^{p}\left(R^{2}\right)$ for $p>1$, see [16, p. 477] or [15].

2) If $\phi(t)=t^{\alpha}$ with $\alpha \in(0,1]$, then $M_{\phi}$ is bounded on $L^{p}\left(R^{2}\right)$ for $p>1$, see [13].

3) If $\phi(t) \in C^{1}([0,1))$ such that $\phi(0)=\phi^{\prime}(0)=0$ and $\phi(t)^{\prime}$ is a convex increasing function for $t>0$, $M_{\phi}$ is bounded on $L^{p}\left(R^{2}\right)$ for $p>1$, see [13], see [7, Corollary 5.3].

4) Let $b(t)=t \phi^{\prime}(t)-\phi(t)$. If $\phi(t) \in C^{2}([0,1))$, $\phi(t)$ is convex on $[0, \infty)$ with $\phi(0)=\phi^{\prime}(0)=0$, and there exists an $\varepsilon>0$ so that for each $t>0$,

$b^{\prime}(t)>\varepsilon b(t) / t$, then $M_{\phi}$ is bounded on $L^{p}\left(R^{2}\right)$ for $p>1$, see [4, Theorem 1.5]. In particular, if $\phi(t)$ is ether even or odd and there exists a $0<C<\infty$ so that for each $t>0, \phi^{\prime}(C t)>2 \phi(t)$, then $M_{\phi}$ is bounded on $L^{p}\left(R^{2}\right)$ for $p>1$, see [3] or [4].
In order to obtain Theorem 1, we let $S_{\Omega, \phi}$ be the operator defined by

$$
\begin{aligned}
& S_{\Omega, \phi}(f)\left(x, x_{n+1}\right) \\
& :=\left(\int_{0}^{\infty}\left|\int_{S^{n-1}} \Omega\left(y^{\prime}\right) f\left(x-r y^{\prime}, x_{n+1}-\phi(|y|)\right) \mathrm{d} \sigma\left(y^{\prime}\right)\right|^{2} \frac{\mathrm{d} r}{r}\right)^{1 / 2} .
\end{aligned}
$$

Clearly, if $h \in L^{2}\left(R^{+}, r^{-1} d r\right)$, then

$$
\left|T_{\phi, h}(f)\left(x, x_{n+1}\right)\right| \leq\|h\|_{L^{2}\left(R^{+}, r^{-1} d r\right)} S_{\Omega, \phi}(f)\left(x, x_{n+1}\right) .
$$

Therefore Theorem 1 can be deduced immediately from the next theorem.

Theorem 2. Let $\phi$ and $\Omega$ be as in Theorem 1 . Then

1) $\left\|S_{\Omega, \phi}(f)\right\|_{L^{2}\left(R^{n+1}\right)} \leq C\|f\|_{L^{2}\left(R^{n+1}\right)}$.

2) $\left\|S_{\Omega, \phi}(f)\right\|_{L^{p}\left(R^{n+1}\right)} \leq C\|f\|_{L^{p}\left(R^{n+1}\right)}$,

$2<p<\infty$, provided that the maximal operator $M_{\phi}$ in (1.3) is bounded on $L^{p}\left(R^{2}\right)$ for $1<p<\infty$.

Remark 2. By the similar arguments as in [1], we remark that the condition $\Omega \in L\left(\log ^{+} L\right)^{1 / 2}\left(S^{n-1}\right)$ is optimal. Precisely, there exists an $\Omega$ lies in

$\Omega \in L\left(\log ^{+} L\right)^{\varepsilon}\left(S^{n-1}\right)$ for all $\varepsilon<1 / 2$ and satisfies (1.1) such that $S_{\Omega, \phi}$ is unbounded on $L^{p}\left(R^{n+1}\right)$. And it is worth pointing out that the size condition is much weaker than that for the classical Calderón-Zygmund singular integral operators.

This paper is organize as follows. In Section 2 we will give the proofs of our theorems. An extension of our main results will be given in Section 3. We would like to remark that the main ideas in the proofs of our results are taken from $[7,9,17]$.

Throughout this paper, we always use letter $C$ to denote positive constants that may vary at each occurrence but are independent of the essential variables.

\section{Proofs of Main Results}

Let us begin with a lemma, which will play a key role in the proofs of our main results.

Lemma 2.1. Let $\Omega \in L^{1}\left(S^{n-1}\right)$ and satisfy (1.1). If the maximal operator $M_{\phi}$ in (1.3) is bounded on $L^{p}\left(R^{2}\right)$ for, then the following maximal operator $M_{\Omega, \phi}$ defined by

$$
\begin{aligned}
& M_{\Omega, \phi}(f)\left(x, x_{n+1}\right) \\
& :=\sup _{R>0} \int_{R / 2<\mid y \leq R} \frac{\left|\Omega\left(y^{\prime}\right)\right|}{|y|^{n}} \times\left|f\left(x+y, x_{n+1}+\phi(|y|)\right)\right| \mathrm{d} y \\
& \text { is bounded on } \quad L^{p}\left(R^{n+1}\right) \text { with bound } \\
& C\|\Omega\|_{L^{1}\left(s^{n-1}\right)}, \quad 1<p<\infty .
\end{aligned}
$$


Proof. Since

$$
\begin{aligned}
& M_{\Omega, \phi}(f)\left(x, x_{n+1}\right) \\
& =\sup _{R>0} \int_{R / 2}^{R} \int_{s^{n-1}}\left|\Omega\left(y^{\prime}\right)\right|\left|f\left(x+r y^{\prime}, x_{n+1}+\phi(r)\right)\right| \mathrm{d} \sigma\left(y^{\prime}\right) \frac{\mathrm{d} r}{r} \\
& \leq \int_{S^{n-1}}\left|\Omega\left(y^{\prime}\right)\right|\left|\sup _{R>0} \int_{R / 2}^{R}\right| f\left(x+r y^{\prime}, x_{n+1}+\phi(r)\right) \mid \frac{\mathrm{d} r}{r} \mathrm{~d} \sigma\left(y^{\prime}\right) \\
& \leq \int_{S^{n-1}}\left|\Omega\left(y^{\prime}\right)\right| M_{\phi, y^{\prime}}(f)\left(x, x_{n+1}\right) \mathrm{d} \sigma\left(y^{\prime}\right)
\end{aligned}
$$

where

$M_{\phi, y^{\prime}}(f)\left(x, x_{n+1}\right):=\sup _{R>0} \frac{1}{R} \int_{R}^{2 R}\left|f\left(x+r y^{\prime}, x_{n+1}+\phi(r)\right)\right| \mathrm{d} r$.

Thus by Minkowski's inequality, we have

$$
\left\|M_{\Omega, \phi}(f)\right\|_{L^{p}\left(R^{n+1}\right)} \leq \int_{S^{n-1}}\left|\Omega\left(y^{\prime}\right)\right|\left\|M_{\phi, y^{\prime}}(f)\right\|_{L^{p}\left(R^{n+1}\right)} \mathrm{d} \sigma\left(y^{\prime}\right) .
$$

It remains to show that

$$
\left\|M_{\phi, y^{\prime}}(f)\right\|_{L^{p}\left(R^{n+1}\right)} \leq C\|f\|_{L^{p}\left(R^{n+1}\right)}
$$

with $C$ independent of $y^{\prime}$.

Let $\mathbf{1}=(1,0, \cdots, 0) \in S^{n-1}$. For each fixed $y^{\prime} \in S^{n-1}$, choose a rotation $\rho$ such that $\rho y^{\prime}=\mathbf{1}$. Denote the inverse of $\rho$ by $\rho^{-1}$ and define the function $f_{\rho}$ by $f_{\rho}\left(x, x_{n+1}\right)=f\left(\rho x, x_{n+1}\right)$. Then

$$
f\left(x+r y^{\prime}, x_{n+1}+\phi(r)\right)=f_{\rho^{-1}}\left(\rho x+r \mathbf{1}, x_{n+1}+\phi(r)\right) .
$$

This together with the $L^{p}$-bondedness of $M_{\phi}$, and change of variables, show that

$$
\left\|M_{\phi, y^{\prime}}(f)\right\|_{L^{p}\left(R^{n+1}\right)} \leq C\|f\|_{L^{p}\left(R^{n+1}\right)}, 1<p<\infty,
$$

where $C$ is independent of. Lemma 2.1 is proved.

Next we introduce some notations. Assume that

$\Omega \in L\left(\log ^{+} L\right)^{1 / 2}\left(S^{n-1}\right)$ and satisfies (1.1). For any $l \in \mathbb{N}$, let $E_{l}=\left\{y^{\prime} \in S^{n-1}: 2^{l} \leq\left|\Omega\left(y^{\prime}\right)\right|<2^{l+1}\right\}$ Also,

we let $E_{0}=\left\{y^{\prime} \in S^{n-1}:\left|\Omega\left(y^{\prime}\right)\right|<2\right\}$ Set

$D=\left\{l \in \mathbb{N}: \quad \sigma\left(E_{l}\right)>2^{-4 l}\right\}$ and for $l \geq 1$,

$$
\begin{array}{r}
\Omega_{l}\left(y^{\prime}\right):=\Omega\left(y^{\prime}\right) \chi_{E_{l}}\left(y^{\prime}\right)-\sigma\left(S^{n-1}\right)^{-1} \\
\int_{S^{n-1}} \Omega\left(y^{\prime}\right) \chi_{E_{l}}\left(y^{\prime}\right) \mathrm{d} \sigma\left(y^{\prime}\right),
\end{array}
$$

and $\Omega_{0}\left(y^{\prime}\right):=\Omega\left(y^{\prime}\right)-\sum_{l \in D} \Omega_{l}\left(y^{\prime}\right)$. Then we have the following:

$$
\begin{gathered}
\int_{S^{n-1}} \Omega_{l}\left(y^{\prime}\right) \mathrm{d} \sigma\left(y^{\prime}\right)=0, l \geq 0, \\
\left\|\Omega_{l}\right\|_{1} \leq 2\left\|\Omega \chi_{E_{l}}\right\|_{1}:=2 A_{l}, l \in D, \\
\left\|\Omega_{0}\right\|_{1} \leq C\left\|\Omega_{0}\right\|_{2} \leq C<\infty,
\end{gathered}
$$

$$
\begin{gathered}
\Omega\left(y^{\prime}\right)=\sum_{l \in D \cup\{0\}} \Omega_{l}\left(y^{\prime}\right), \\
\sum_{l \in D \cup\{0\}}(l+1)^{1 / 2} A_{l} \leq C\|\Omega\|_{L\left(\log ^{+} L\right)^{1 / 2}\left(s^{n-1}\right)},
\end{gathered}
$$

where $A_{l}=\left\|\Omega \chi_{E_{l}}\right\|_{1}$ for $l \in D$ and $A_{0}=1$.

Now we give the proofs of our theorems as follows.

Proof of Theorem 2. For each $l \in D \cup\{0\}$, we let

$S_{\Omega_{l}, \phi}(f)\left(x, x_{n+1}\right):=$

$\left(\int_{0}^{\infty}\left|\int_{S^{n-1}} \Omega_{l}\left(y^{\prime}\right) f\left(x-r y^{\prime}, x_{n+1}-\phi(|y|)\right) \mathrm{d} \sigma\left(y^{\prime}\right)\right|^{2} \frac{\mathrm{d} r}{r}\right)^{1 / 2}$.

By (2.5) and Minkowski's inequality, we have

$$
S_{\Omega, \phi}(f)\left(x, x_{n+1}\right) \leq \sum_{l \in D \cup\{0\}} S_{\Omega_{l}, \phi}(f)\left(x, x_{n+1}\right) .
$$

For any $l \in D \cup\{0\}$, let's argue as in the proof of Theorem 2.1 in [1], choose a collection of $C^{\infty}$ function $\left\{\psi_{l, j}\right\}_{j \in \mathbb{Z}}$ on $(0, \infty)$ with the following properties:

$$
\begin{aligned}
& \operatorname{supp}\left(\psi_{l, j}\right) \subseteq\left[2^{-(j+1)(l+1)}, 2^{-(j-1)(l+1)}\right], \\
& 0 \leq \psi_{l, j}(t) \leq 1, \text { and } \sum_{j \in \mathbb{Z}}\left[\psi_{l, j}(t)\right]^{2}=1, \\
& \\
&\left|\frac{\mathrm{d}^{\alpha} \psi_{l, j}(t)}{\mathrm{d} t^{\alpha}}\right| \leq \frac{C_{\alpha}}{t^{\alpha}},
\end{aligned}
$$

where $t>0, \quad \alpha \in \mathbb{N}$, and $C_{\alpha}$ is a constant independent of $l$.

For each $j \in \mathbb{Z}$ and $l \in D \cup\{0\}$, denote by $S_{l, j}$ the multiplier

$$
\widehat{S_{l, j}}(f)\left(\xi, \xi_{n+1}\right)=\psi_{l, j}(|\xi|) \widehat{f}\left(\xi, \xi_{n+1}\right),
$$

and $S_{l, j}^{2}$ by

$$
S_{l, j}^{2}(f)\left(x, x_{n+1}\right)=S_{l, j}\left(S_{l, j}(f)\right)\left(x, x_{n+1}\right) .
$$

Then by (2.9) and Minkowski's inequality,

$$
\begin{aligned}
& S_{\Omega_{l}, \phi}(f)\left(x, x_{n+1}\right) \\
& =\left(\sum_{j \in \mathbb{Z}} \int_{2^{j}}^{2^{(j+1)(l+1)}} \mid \sum_{k \in \mathbb{Z}} \int_{S^{n-1}} \Omega_{l}\left(y^{\prime}\right)\right. \\
& \left.\times\left. S_{l, j+k}^{2}(f)\left(x-r y^{\prime}, x_{n+1}-\phi(r)\right) \mathrm{d} \sigma\left(y^{\prime}\right)\right|^{2} \frac{\mathrm{d} r}{r}\right)^{1 / 2} \\
& \leq \sum_{k \in \mathbb{Z}}\left(\sum_{j \in \mathbb{Z}} \int_{1}^{2^{l+1}} \mid \int_{S^{n-1}} \Omega_{l}\left(y^{\prime}\right)\right. \\
& \left.\times\left. S_{l, j+k}^{2}(f)\left(x-r y^{\prime}, x_{n+1}-\phi(r)\right) \mathrm{d} \sigma\left(y^{\prime}\right)\right|^{2} \frac{\mathrm{d} r}{r}\right)^{1 / 2} \\
& :=\sum_{k \in \mathbb{Z}} I_{l, k}(f)\left(x, x_{n+1}\right),
\end{aligned}
$$

where 


$$
\begin{aligned}
& I_{l, k}(f)\left(x, x_{n+1}\right)=\left(\sum_{j \in \mathbb{Z}} \int_{1}^{2^{l+1}} \mid \int_{S^{n-1}} \Omega_{l}\left(y^{\prime}\right)\right. \\
& \left.\times\left. S_{l, j+k}^{2}(f)\left(x-r y^{\prime}, x_{n+1}-\phi(r)\right) \mathrm{d} \sigma\left(y^{\prime}\right)\right|^{2} \frac{\mathrm{d} r}{r}\right)^{1 / 2} .
\end{aligned}
$$

This together with (2.7) implies

$$
\left\|S_{\Omega, \phi}(f)\right\|_{L^{p}\left(R^{n+1}\right)} \leq \sum_{l \in D \cup\{0\}} \sum_{k \in \mathbb{Z}}\left\|I_{l, k}(f)\right\|_{L^{p}\left(R^{n+1}\right)} .
$$

Now we estimate $\left\|I_{l, k}(f)\right\|_{L^{p}\left(R^{n+1}\right)}$ in the following
ases:

Case 1. For $p=2$, we claim that there exists $\delta>0$ such that for $l \in D \cup\{0\}$,

$$
\left\|I_{l, k}(f)\right\|_{L^{2}\left(R^{n+1}\right)} \leq C 2^{-\delta|k|}(l+1)^{1 / 2} A_{l}\|f\|_{L^{2}\left(R^{n+1}\right)},
$$

where $C$ is independent of $l$ and $k$.

Indeed, by Plancheral's theorem and Fubini's theorem,

$$
\left\|I_{l, k}(f)\right\|_{2}^{2} \leq \sum_{j \in \mathbb{Z}} \int_{\Delta_{l, j+k}}\left|\widehat{f}\left(\xi, \xi_{n+1}\right)\right|^{2} \times J_{l, j}(\xi) \mathrm{d} \xi \mathrm{d} \xi_{n+1},
$$

where

$$
\begin{gathered}
\Delta_{l, j+k}:=\left\{\xi \in R^{n}: \quad 2^{-(j+k+l)(l+1)} \leq|\xi| \leq 2^{-(j+k-1)(l+1)}\right\} \times R, \\
J_{l, j}(\xi)=\int_{1}^{2^{l+1}}\left|\int_{S^{n-1}} \Omega_{l}\left(y^{\prime}\right) \mathrm{e}^{-i 2^{j(l+1)} r y^{\prime} \bullet \xi} \mathrm{d} \sigma\left(y^{\prime}\right)\right|^{2} \frac{\mathrm{d} r}{r}
\end{gathered}
$$

In order to prove (2.12), we first estimate $J_{l, j}(\xi)$. Obviously, we have

$$
J_{l, j}(\xi) \leq(l+1)\left\|\Omega_{l}\right\|_{1}^{2} \leq C(l+1) A_{l}^{2} .
$$

By (2.2), a straightforward computing shows that

$$
\begin{aligned}
J_{l, j}(\xi) & \leq(l+1)\left\|\Omega_{l}\right\|_{1}^{2}\left|2^{(j+1)(l+1)} \xi\right|^{2} \\
& \leq C(l+1) A_{l}^{2}\left|2^{(j+1)(l+1)} \xi\right|^{2}
\end{aligned}
$$

Using interpolation between (2.14) and (2.15), we get

$$
J_{l, j}(\xi) \leq C(l+1) A_{l}^{2}\left|2^{(j+1)(l+1)} \xi\right|^{2 /(l+1)} .
$$

On the other hand, we have

$$
\begin{aligned}
J_{l, j}(\xi)= & \int_{1}^{2^{l+1}} \iint_{S^{n-1} \times S^{n-1}} \Omega_{l}\left(y^{\prime}\right) \overline{\Omega_{l}\left(u^{\prime}\right)} \\
& \times \mathrm{e}^{-i 2^{j(l+1)} r\left(y^{\prime}-u\right) \bullet \xi} \mathrm{d} \sigma\left(y^{\prime}\right) \mathrm{d} \sigma\left(u^{\prime}\right) \frac{\mathrm{d} r}{r} \\
= & \iint_{S^{n-1} \times S^{n-1}} \Omega_{l}\left(y^{\prime}\right) \overline{\Omega_{l}\left(u^{\prime}\right)} \\
& \times \int_{1}^{2^{l+1}} \mathrm{e}^{-i 2^{j(l+1)} r\left(y^{\prime}-u\right) \bullet \xi} \frac{\mathrm{d} r}{r} \mathrm{~d} \sigma\left(y^{\prime}\right) \mathrm{d} \sigma\left(u^{\prime}\right)
\end{aligned}
$$

And by integration by parts,

$$
\begin{aligned}
& \left|\int_{1}^{2^{l+1}} \mathrm{e}^{-i 2^{j(l+1)} r\left(y^{\prime}-u\right) \cdot \xi} \frac{\mathrm{d} r}{r}\right| \\
& \leq C(l+1)\left|2^{j(l+1)}\left(y^{\prime}-u^{\prime}\right) \bullet \xi\right|^{-1},
\end{aligned}
$$

with the easy fact

$$
\left|\int_{1}^{2^{l+1}} \mathrm{e}^{-i 2^{j(l+1)} r\left(y^{\prime}-u\right) \bullet \xi} \frac{\mathrm{d} r}{r}\right| \leq l+1,
$$

we obtain

$$
\begin{aligned}
& \left|\int_{1}^{2^{l+1}} \mathrm{e}^{-i 2^{j(l+1)} r\left(y^{\prime}-u\right) \bullet \xi} \frac{\mathrm{d} r}{r}\right| \\
& \leq C(l+1)\left|2^{j(l+1)}\left(y^{\prime}-u^{\prime}\right) \bullet \xi\right|^{-1 / 4}
\end{aligned}
$$

Thus, by Holder's inequality

$$
\begin{aligned}
& J_{l, j}(\xi) \leq C(l+1)\left|2^{j(l+1)} \xi\right|^{-1 / 4}\left\|\Omega_{l}\right\|_{2}^{2} \\
& \left\{\iint_{S^{n-1} \times S^{n-1}} \times\left|\left(y^{\prime}-u^{\prime}\right) \bullet \xi^{\prime}\right|^{-1 / 2} d \sigma\left(y^{\prime}\right) d \sigma\left(u^{\prime}\right)\right\}^{1 / 2} \\
& \leq C(l+1)\left|2^{j(l+1)} \xi\right|^{-1 / 4}\left\|\Omega_{l}\right\|_{2}^{2} .
\end{aligned}
$$

Note that $\left\|\Omega_{0}\right\|_{2} \leq C=C A_{0}$, and for $l \in D$, $A_{l} \leq C 2^{l} \sigma\left(E_{l}\right) \leq C 2^{-3 l}$, we have

$$
\left\|\Omega_{l}\right\|_{2} \leq C 2^{l+1} \sigma\left(E_{l}\right)^{1 / 2} \leq C 2^{2(l+1)} A_{l} .
$$

Consequently,

$$
J_{l, j}(\xi) \leq C(l+1) A_{l}^{2} 2^{4(l+1)}\left|2^{j(l+1)} \xi\right|^{-1 / 4} .
$$

This together with (2.14) and an interpolation implies

$$
J_{l, j}(\xi) \leq C(l+1) A_{l}^{2}\left|2^{j(l+1)} \xi\right|^{-1 / 4(l+1)} .
$$

Then by the fact that $\Delta_{l, j+k} \cap \Delta_{l, j^{\prime}+k}=\varnothing$ whenever $j^{\prime} \notin\{j-1, j, j+1\}$, (2.12) follows from (2.13), (2.16) and (2.17).

Case 2. For $p>2$, we shall show that there exists $\theta>0$ such that for $l \in D \cup\{0\}$,

$$
\left\|I_{l, k}(f)\right\|_{L^{p}\left(R^{n+1}\right)} \leq C 2^{-\theta|k|}(l+1)^{1 / 2} A_{l}\|f\|_{L^{p}\left(R^{n+1}\right)} .
$$

where $C$ is independent of $l$ and $k$.

Indeed, choose $g \in L^{(p / 2)^{\prime}}\left(R^{n+1}\right)$ such that $\|g\|_{L^{(p / 2)}\left(R^{n+1}\right)}=1$ and

$$
\begin{aligned}
& \left\|I_{l, k}(f)\right\|_{L^{p}\left(R^{n+1}\right)}^{2}=\int_{R^{n+1}} \sum_{j \in \mathbb{Z}} \int_{1}^{2^{l+1}} \mid \int_{S^{n-1}} \Omega_{l}\left(y^{\prime}\right) \\
& \times\left. S_{l, j+k}^{2}(f)\left(x-2^{j(l+1)} r y^{\prime}, x_{n+1}-\phi\left(2^{j(l+1)} r\right)\right) \mathrm{d} \sigma\left(y^{\prime}\right)\right|^{2} \\
& \times \frac{\mathrm{d} r}{r} g\left(x, x_{n+1}\right) \mathrm{d} x \mathrm{~d} x_{n+1} .
\end{aligned}
$$

By Holder's inequality, we get 


$$
\begin{aligned}
& || I_{l, k}(f) \|_{p}^{2} \leq \int_{R^{n+1}} \sup _{j \in \mathbb{Z}} \int_{1}^{2^{l+1}} \int_{S^{n-1}}\left|\Omega_{l}\left(y^{\prime}\right)\right| \\
& \times\left|g\left(x+2^{j(l+1)} r y^{\prime}, x_{n+1}+\phi\left(2^{j(l+1)} r\right)\right)\right| \mathrm{d} \sigma\left(y^{\prime}\right) \frac{\mathrm{d} r}{r} \\
& \times \sum_{j \in \mathbb{Z}}\left|S_{l, j+k}^{2}(f)\left(x, x_{n+1}\right)\right|^{2} \mathrm{~d} x \mathrm{~d} x_{n+1}\left\|\Omega_{l}\right\|_{1} .
\end{aligned}
$$

Note that

$$
\begin{aligned}
& \sup _{j \in \mathbb{Z}} \int_{1}^{2^{l+1}} \int_{S^{n-1}}\left|\Omega_{l}\left(y^{\prime}\right)\right| \\
& \times\left|g\left(x+2^{j(l+1)} r y^{\prime}, x_{n+1}+\phi\left(2^{j(l+1)} r\right)\right)\right| \mathrm{d} \sigma\left(y^{\prime}\right) \frac{\mathrm{d} r}{r} \\
& \leq(l+1) M_{\Omega_{l}, \phi}(g)\left(x, x_{n+1}\right) .
\end{aligned}
$$

Applying Holder's inequality again, it follows from Lemma 2.1 and the Littlewood-Paley theory (see [18, Chapter 4]) that

$$
\begin{aligned}
& \left\|I_{l, k}(f)\right\|_{p}^{2} \leq C(l+1)\left\|\Omega_{l}\right\|_{1}^{2}\|g\|_{(p / 2)^{\prime}} \\
& \times\left\|\left(\sum_{j \in \mathbb{Z}}\left|S_{l, j+k}^{2}(f)\right|^{2}\right)^{1 / 2}\right\| \\
& \leq C(l+1)\left\|\Omega_{l}\right\|_{1}^{2}\left\|\left(\sum_{j \in \mathbb{Z}}\left|S_{l, j+k}^{2}(f)\right|^{2}\right)^{1 / 2}\right\|_{p} \\
& \leq C(l+1)\left\|\Omega_{l}\right\|_{1}^{2}\|f\|_{p}^{2} \leq C(l+1) A_{l}^{2}\|f\|_{p}^{2} .
\end{aligned}
$$

This together with (2.12) and an interpolation implies (2.18).

Therefore, by (2.11), (2.12) and (2.6), we get

$$
\begin{aligned}
& \left\|S_{\Omega, \phi}(f)\right\|_{L^{2}\left(R^{n+1}\right)} \leq C \sum_{l \in D \cup\{0\}} \sum_{k \in \mathbb{Z}}(l+1)^{1 / 2} \\
& \times A_{l} 2^{-\delta|k|}\|f\|_{L^{2}\left(R^{n+1}\right)} \\
& \leq C \sum_{l \in D \cup\{0\}}(l+1)^{1 / 2} A_{l}\|f\|_{L^{2}\left(R^{n+1}\right)} \\
& \leq C\|\Omega\|_{L\left(\log ^{+} L\right)^{1 / 2}\left(S^{n-1}\right)}\|f\|_{L^{2}\left(R^{n+1}\right)} .
\end{aligned}
$$

This prove 1) of Theorem 2 .

If $M_{\phi}$ is bounded on $L^{p}\left(R^{2}\right)$ for $1<p<\infty$, then by (2.11), (2.18) and (2.6), we obtain that for $p>2$,

$$
\left\|S_{\Omega, \phi}(f)\right\|_{L^{p}\left(R^{n+1}\right)} \leq C\|\Omega\|_{L\left(\log ^{+} L\right)^{1 / 2}\left(S^{n-1}\right)}\|f\|_{L^{p}\left(R^{n+1}\right)},
$$

which completes the proof of Theorem 2 .

Proof of Theorem 1. By the fact

$$
\left|T_{\phi, h}(f)\left(x, x_{n+1}\right)\right| \leq\|h\|_{L^{2}\left(R^{+}, r^{-1} d r\right)} S_{\Omega, \phi}(f)\left(x, x_{n+1}\right),
$$

and Theorem 2, it follows that $T_{\phi, h}$ is bounded on $L^{p}\left(R^{n+1}\right)$ for $2 \leq p<\infty$. On the other hand, by duality we can obtain that $T_{\phi, h}$ is bounded on $L^{p}\left(R^{n+1}\right)$ for $1<p<2$. Theorem 1 is proved.

\section{Further Results}

In this section, we will extend the definition of $T_{\phi, h}$ to higher dimensional cases. Let $\Phi(t)=\left(\phi_{1}(t), \cdots, \phi_{m}(t)\right)$ be a curve on $R^{m}, m \geq 2$, where each $\phi_{i}(t)$ is a real-valued continuous function. For $x, y \in R^{n}$, and $x^{*} \in R^{m}$, we define

$$
\begin{aligned}
& T_{\Phi, h}(f)\left(x, x^{*}\right)=p \cdot v \cdot \int_{R^{n}} h(|y|)|y|^{-n} \Omega\left(y^{\prime}\right) \\
& \times f\left(x-y, x^{*}-\Phi(|y|)\right) \mathrm{d} y .
\end{aligned}
$$

Also, we define the operator $S_{\Omega, \Phi}$ by

$$
\begin{aligned}
& S_{\Omega, \Phi}(f)\left(x, x^{*}\right)=\left(\int_{0}^{\infty} \mid \int_{S^{n-1}} \Omega\left(y^{\prime}\right)\right. \\
& \left.\times\left. f\left(x-r y^{\prime}, x^{*}-\Phi(r)\right) \mathrm{d} \sigma\left(y^{\prime}\right)\right|^{2} \frac{\mathrm{d} r}{r}\right)^{1 / 2},
\end{aligned}
$$

and the lower dimensional maximal function $M_{\Phi}$ by

$$
\begin{aligned}
& M_{\Phi}(f)\left(x_{1}, x_{*}\right) \\
& =\sup _{r>0} r^{-1} \int_{r / 2}^{r}\left|f\left(x_{1}-t, x_{*}-\Phi(t)\right)\right| \mathrm{d} t .
\end{aligned}
$$

In [10], Fan and Zheng extended the result of Theorem B to the operator $T_{\Phi, h}$ for $h \in \Delta_{\gamma}\left(R^{+}\right)$for $\gamma>1$. Here, we will obtain the following results.

Theorem 3. Suppose that $h \in L^{2}\left(R^{+}, r^{-1} d r\right)$ and $\Omega \in$ $L\left(\log ^{+} L\right)^{1 / 2}\left(S^{n-1}\right)$. Then

1) $\left\|T_{\Phi, h}(f)\right\|_{L^{2}\left(R^{n+m}\right)} \leq C\|f\|_{L^{2}\left(R^{n+m}\right)}$

2) $\left\|T_{\Phi, h}(f)\right\|_{L^{p}\left(R^{n+m}\right)} \leq C\|f\|_{L^{p}\left(R^{n+m}\right)}, 1<p<\infty$,

provided that the maximal operator $M_{\Phi}$ defined in (4.3) is bounded on $L^{p}\left(R^{m+1}\right)$ for all $p>1$.

Theorem 4. Let $\Phi$ and $\Omega$ be as in Theorem 3. Then

1) $\left\|S_{\Omega, \Phi}(f)\right\|_{L^{2}\left(R^{n+m}\right)} \leq C\|f\|_{L^{2}\left(R^{n+m}\right)}$.

2) $\left\|S_{\Omega, \Phi}(f)\right\|_{L^{p}\left(R^{n+m}\right)} \leq C\|f\|_{L^{p}\left(R^{n+m}\right)}, \quad 2<p<\infty$,

provided that the maximal operator $M_{\Phi}$ defined in (4.3) is bounded on $L^{p}\left(R^{m+1}\right)$ for all $p>1$.

Clearly, if $m=1$ then Theorem 3 and 4 are reduced to Theorem 1 and 2. For $m \geq 2$, by the same arguments as in the proof of Lemma 2.1, we can obtain the following lemma.

Lemma 4.1. Let $\Omega \in L^{1}\left(S^{n-1}\right)$ and satisfy (1.1). If the maximal operator $M_{\Phi}$ in (4.3) is bounded on $L^{p}\left(R^{m+1}\right)$ for $p>1$, then the following maximal operator $M_{\Omega, \Phi}$ defined by

$$
\begin{aligned}
& M_{\Omega, \Phi}(f)\left(x, x_{*}\right) \\
& :=\sup _{r>0} \int_{r / 2<y \mid \leq r} \frac{\left|\Omega\left(y^{\prime}\right)\right|}{|y|^{n}} \times\left|f\left(x+y, x_{*}+\Phi(|y|)\right)\right| d y
\end{aligned}
$$

is bounded on $L^{p}\left(R^{n+m}\right)$ with bound $C\|\Omega\|_{L^{1}\left(S^{n-1}\right)}$, 
$1<p<\infty$.

Then Theorem 3 and 4 follow from this lemma with the arguments and the estimates similar to those in the proofs of our theorems in Section 2. The details are omitted.

\section{Acknowledgements}

The authors are grateful to the referee for his/her helpful comments and suggestions.

\section{References}

[1] A. Al-Salman and Y. Pan, "Singular Integrals with Rough Kernels in $\mathrm{L}\left(\log ^{+} \mathrm{L}\right)^{1 / 2}\left(\mathrm{~S}^{\mathrm{n}-1}\right)$," Journal of the London Mathematical Society, Vol. 66, No. 2, 2002, pp. 153-174. doi:10.1112/S0024610702003241

[2] H. Al-Qassem and Y. Pan, "Singular Integrals along Surfaces of Revolution with Rough Kernels," SUT Journal of Mathematics, Vol. 39, No. 1, 2003, pp. 55-70.

[3] H. Carlsson, M. Christ, A. Cordoba, J. Duoandikoetxea, J. L. Rubio de Francia, J. Vance, S. Wainger and D. Weinberg, " $\mathrm{L}^{\mathrm{p}}$ Estimates for Maximal Functions and Hilbert Transforms along Flat Convex Plane Curves in $\mathrm{R}^{2}$," Bulletin of the American Mathematical Society, Vol. 14, No. 2, 1986, pp. 263-267. doi:10.1090/S0273-0979-1986-15433-9

[4] A. Carbery, M. Christ, J. Vance, S. Wainger and D. K. Watson, "Operators Associated to Flat Plane Curves: $\mathrm{L}^{\mathrm{p}}$ Estimates via Dilation Methods," Duke Mathematical Journal, Vol. 59, 1989, pp. 675-700. doi:10.1215/S0012-7094-89-05930-9

[5] L. Chen and D. Fan, "On Singular Integrals along Surfaces Related to Block Spaces," Integral Equations and Operator Theory, Vol. 29, 1997, pp. 261-268. doi:10.1007/BF01320700

[6] L. C. Cheng and Y. Pan, " $L^{p}$ Bounds For singular Integrals Associated to Surfaces of Revolution," Journal of Mathematical Analysis and Applications, Vol. 265, 2002, pp. 163-169. doi:10.1006/jmaa.2001.7710

[7] J. Duoandikoetxea and J. L. Rubio de Francia, "Maximal and Singular Integral Operators via Fourier Transform Estimates," Inventiones Mathematicae, Vol. 84, No. 3, 1986, pp. 541-561. doi:10.1007/BF01388746
[8] D. Fan and Y. Pan, "Singular Integral Operators with Rough Kernels Supported by Subvarieties," American Journal of Mathematics, Vol. 119, No. 4, 1997, pp. 799 839. doi:10.1353/ajm.1997.0024

[9] D. Fan and Q. Zheng, "Maximal Singular Integral Operators along Surfaces," Journal of Mathematical Analysis and Applications, Vol. 267, No. 2, 2002, pp. 746-759. doi:10.1006/jmaa.2001.7812

[10] W. Kim, S. Wainger, J. Wright and S. Ziesler, "Singular Integrals and Maximal Functions Associated to Surfaces of Revolution," Bulletin London Mathematical Society, Vol. 28, No. 3, 1996, pp. 291-296. doi:10.1112/blms/28.3.291

[11] S. Lu, Y. Pan and D. Yang, "Rough Singular Integrals Associated to Surfaces of Revolution," Proceedings of the AMS-American Mathematical Society, Vol. 129, 2001, pp. 2931-2940. doi:10.1090/S0002-9939-01-05893-2

[12] Y. Pan, L. Tang and D. Yang, "Boundedness of Rough Singular Integrals Associated with Surfaces of Revolution," Advances in Mathematics (China), Vol. 32, No. 2, 2003, pp. 677-682.

[13] F. Ricci and E. M. Stein, "Multiparameter Singular Integrals and Maximal Functions," Annales de l'institut Fourier, Vol. 42, No. 3, 1992, pp. 637-670. doi:10.5802/aif.1304

[14] E. M. Stein, "Problem in Harmonic Analysis Related to Curvature and Oscillatory Integrals," Proceedings of the International Conference on Mathematics, Berkely, 1986, pp. 196-221.

[15] E. M. Stein and S. Wainger, "Problems in Harmonic Analysis Related to Curvature," Bulletin of the American Mathematical Society, Vol. 84, No. 6, 1978, pp. 12391295.

[16] E. M. Stein, "Harmonic Analysis: Real-Variable Methods, Orthogonality and Oscillatory Integrals," Princeton University Press, Princeton, 1993. doi:10.1090/S0002-9904-1978-14554-6

[17] A. Al-Salman, "On Maximal Functions with Rough Kernels in $\mathrm{L}\left(\log ^{+} \mathrm{L}\right)^{1 / 2}\left(\mathrm{~S}^{\mathrm{n}-1}\right)$, , Collectanea Mathematica, Vol. 56, No. 1, 2005, pp. 47-56.

[18] E. M. Stein, "Singular Integrals and Differentiability Properties of Functions," Princeton University Press, Princeton, 1970. 